\title{
FLAT COVERS AND FLAT COTORSION MODULES
}

\author{
EDGAR ENOCHS
}

\begin{abstract}
It is not known whether modules over an arbitrary ring have flat covers, however for certain modules over commutative noetherian rings they can be shown to exist. These covers, in turn, have an interesting connection with flat cotorsion modules. A complete description of flat cotorsion modules analogous to that given by Harrison for torsion free, cotorsion abelian groups will be given.

In this article, $R$ will denote a commutative noetherian ring.
\end{abstract}

NotAtion. If $R$ is a local ring, $m(R)$ will denote its maximal ideal. For $p \in \operatorname{Spec}(R), \hat{R}_{p}$ will denote the completion of the local ring $R_{p}$, and for any $R$-module, $\hat{M}_{p}$ will denote the (separated) completion of the $R_{p}$ module $M_{p}$ with the $m\left(R_{p}\right)$-adic topology. $k(p)$ denotes the residue field of $R_{p}\left(\cong \hat{R}_{p} / m\left(\hat{R}_{p}\right)\right) . E(M)$ will be an injective envelope of $M$. If $R$ is local, $M^{\nu}$ denotes the Matlis dual, $\operatorname{Hom}(M, E(R / m(R)))$, of $M$, and $M$ is said to be reflexive if $M^{\nu \nu} \cong M$ naturally. For a set $X, M^{X}$ is the module of all functions $X \rightarrow M$ and $M^{(X)}$ the submodule of those functions with finite support. $\operatorname{Soc}(M)$ will denote the socle of $M$.

\section{Flat covers.}

Definition (SEe [1]). A linear map $\phi: F \rightarrow M$ is said to be a flat precover of $M$ if $F$ is flat and if any diagram

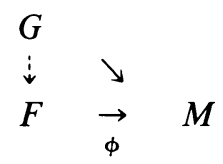

where $G$ is flat can be completed to a commutative diagram (i.e. $\operatorname{Hom}(G, F) \rightarrow$ $\operatorname{Hom}(G, M)$ is surjective). If, furthermore

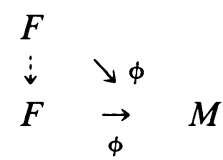

can only be completed by automorphisms of $F$, we say $F$ is a flat cover of $M$ (with $\phi$ understood). If $M$ has a flat precover then it has a cover [1, Theorem 3.1]. If it has a cover, it is clearly unique. If $R$ is Prüfer they always exist and coincide with the torsion free covers shown to exist in [2, Theorems 1,2]. For example, if $k$ is a field, $k[[x]] \rightarrow k$ (evaluation at 0 ) is a flat and torsion free cover of the $k[x]$-module $k$

Received by the editors October 27, 1983.

1980 Mathematics Subject Classification. Primary 13C05, 13C11.

C1984 American Mathematical Society $0002-9939 / 84 \$ 1.00+\$ .25$ per page 
(with $x k=0$ ) (see [2 or 3]). If $n \geqslant 2$, then $k\left[\left[x_{1}, \ldots, x_{n}\right]\right] \rightarrow k$, as noted in [4], is not a torsion free cover, but will be shown to be a flat cover below.

If $\phi^{\prime}: F^{\prime} \rightarrow M$ is a flat precover, $\phi: F \rightarrow M$ a flat cover and $\phi \circ f=\phi^{\prime}$, then $f$ is surjective and $\operatorname{Ker}(f)$ is a direct summand of $F^{\prime}$. Hence we have:

LEMMA 1.1. A flat precover $\phi: F \rightarrow M$ is a cover if and only if $\operatorname{Ker}(\phi)$ contains no nonzero direct summand of $F$.

The following are easy:

LEMMA 1.2. $M=\prod M_{i}$ has a flat cover if and only if each $M_{j}$ does.

Proof. If $F_{i} \rightarrow M_{i}$ is a flat cover for each $i$ then $\prod F_{k} \rightarrow \prod M_{i}$ is a flat precover, so, as noted above, $\Pi M_{i}$ has a flat cover. If $F \rightarrow \Pi M_{i}$ is a flat cover then for any $j$, $F \rightarrow \Pi M_{i} \rightarrow M_{j}$ is a flat precover, so $M_{j}$ has a cover.

LEMMA 1.3. If $\phi: F \rightarrow M$ is a flat cover and $F=F_{1} \oplus F_{2}, M=M_{1} \oplus M_{2}$ are decompositions compatible with $\phi$ (i.e. $\left.\phi\left(F_{i}\right) \subset M_{i}\right)$, then $F_{i} \rightarrow M_{i}$ is a flat cover for $i=1,2$.

Proof. Easily $F_{i} \rightarrow M_{i}$ is a precover. By Lemma 1.1, it is also a cover.

REMARK. If each $F_{i} \rightarrow M_{i}$ is a flat cover and the index set $I$ is infinite, $\Pi F_{i} \rightarrow \Pi M_{i}$ may fail to be a cover and $\oplus F_{i} \rightarrow \oplus M_{i}$ may fail to be a precover. If $I$ is finite, $\oplus F_{i} \rightarrow \oplus M_{i}$ is a cover.

In the following we will need

LEMMA 1.4 (ISHIKAWA [5, THEOREM 1.5]). If $E$ and $E^{\prime}$ are injective modules, then $\operatorname{Hom}\left(E, E^{\prime}\right)$ is a flat $R$-module.

Proposition 1.1. If $M$ is any module and $E$ is an injective module, then $\operatorname{Hom}(E, M)$ and $\operatorname{Hom}(M, E)$ have flat covers.

Proof. From $M \subset E(M)$ we have a map $\operatorname{Hom}(E(M), E) \rightarrow \operatorname{Hom}(M, E)$. But $\operatorname{Hom}(E(M), E)$ is flat, so we want to show that if $F$ is flat, $\operatorname{Hom}(F, \operatorname{Hom}(E(M), E)) \rightarrow \operatorname{Hom}(F, \operatorname{Hom}(M, E))$ is a surjection, or, equivalently, that $\operatorname{Hom}(F \otimes E(M), E) \rightarrow \operatorname{Hom}(F \otimes M, E)$ is, but this is obvious, $F$ being flat and $E$ injective. Since $\operatorname{Hom}(M, E)$ has a flat precover, it has a flat cover.

The case $\operatorname{Hom}(E, M)$ is similar. Here we use the fact that $M$ has an injective precover $E^{\prime} \rightarrow M$ (i.e. $\operatorname{Hom}\left(E, E^{\prime}\right) \rightarrow \operatorname{Hom}(E, M)$ is surjective for any injective module $E$ ) (see [1, Proposition 2.2]). Then if $F$ is flat, to $\operatorname{show} \operatorname{Hom}\left(F, \operatorname{Hom}\left(E, E^{\prime}\right)\right)$ $\rightarrow \operatorname{Hom}(F, \operatorname{Hom}(E, M))$ (or, equivalently, $\left.\operatorname{Hom}\left(F \otimes E, E^{\prime}\right) \rightarrow \operatorname{Hom}(F \otimes E, M)\right)$ is a surjection, we need only note that $F \otimes E$ is injective.

Proposition 1.2. If $M$ is an $\hat{R}_{p}$-module and Matlis reflexive, then $M$ has a flat cover as an $R$-module.

Proof. Since $M \cong M^{\nu v}=\operatorname{Hom}\left(M^{\nu}, E(k(p))\right), M$ has a flat $\hat{R}_{p}$-module cover $F \rightarrow M$ by Proposition 1.1. If $G$ is a flat $R$-module and $G \rightarrow M$ is linear, we have a factorization $G \rightarrow G \otimes \hat{R}_{p} \rightarrow M$. But $G \otimes \hat{R}_{p}$ is $\hat{R}_{p}$ flat, so $G \otimes \hat{R}_{p} \rightarrow M$ can be 
lifted to $F$. Hence $F \rightarrow M$ is a flat precover as $R$-modules since $F$ is flat as an $R$-module.

If $R$ is a complete local ring, finitely generated and artinian modules are known to be reflexive. If $S \subset M$ then the diagram

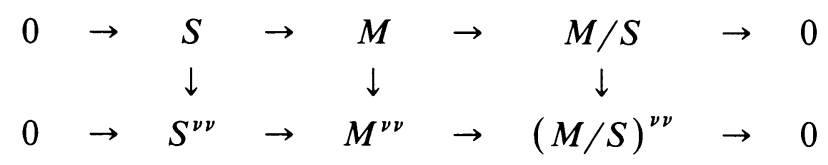

with exact rows and injective vertical maps shows that $M$ is reflexive if and only if $S$ and $M / S$ are. A direct sum $\oplus M_{i}$ is reflexive if and only if each $M_{i}$ is reflexive and $M_{i}=0$ except for a finite number of $i$. This gives an easy characterization of reflexive modules (which may be known, but I have not located it in the literature).

Proposition 1.3. If $R$ is a complete local ring then $M$ is Matlis reflexive if and only if $M$ has a finitely generated submodule $S$ such that $M / S$ is artinian.

Proof. The "only if" follows from the above. If $M=0$ there is nothing to prove. If $M \neq 0$ there is a finitely generated submodule $S_{1} \subset M$ such that $\operatorname{Soc}\left(M / S_{1}\right) \neq 0$. If $\operatorname{Soc}\left(M / S_{1}\right)$ is essential in $M / S_{1}$, it is well known that $M / S_{1}$ (and in fact $\left.E\left(M / S_{1}\right)\right)$ is artinian. If it is not essential, let $N / S_{1} \cap \operatorname{Soc}\left(M / S_{1}\right)=0$ with $S_{1} \subsetneq N$. Then there is a finitely generated $S_{2}$ with $S_{1} \subset S_{2} \subset N$ and $\operatorname{Soc}\left(N / S_{2}\right) \neq 0$. But then $\operatorname{Soc}\left(M / S_{1}\right) \rightarrow \operatorname{Soc}\left(M / S_{2}\right)$ is injective but not surjective. We repeat the procedure and see that it must stop, for otherwise if $T=\bigcup S_{n}, \operatorname{Soc}(M / T)$ is an infinite direct sum. This is not possible by the above.

EXAMPLE. Since $k(p)$ is a reflexive $\hat{R}_{p}$-module, it has a flat cover over $R$. Hence for any set $X, k(p)^{X}$ and, hence, $k(p)^{(X)}$ (it is a direct summand) has a flat cover over $R$.

Using the proof of Proposition 1.1 we see that a precover of $k(p)^{(X)} \cong$ $\operatorname{Hom}\left(k(p), E(k(p))^{(X)}\right)$ is $\operatorname{Hom}\left(E(k(p)), E(k(p))^{(X)}\right)$. As noted in Griffith [7, p. 306, and also in Fuchs 8, Proposition 44.3, in the case $R=Z$ ] the latter module is the completion of a free $\hat{R}_{p}$-module with base indexed by $X$, i.e. it is the submodule of $\hat{R}_{p}^{X}$ consisting of elements $\left(r_{x}\right)$ with countable support and such that $\lim r_{x_{i}}=0$ for any distinct $x_{1}, x_{2}, x_{3}, \ldots$ in $X$. If $T$ designates this module then $T \rightarrow k(p)^{(X)}$ is a flat precover and is seen to induce an isomorphism $T / m\left(\hat{R}_{p}\right) T \rightarrow k(p)^{(X)}$. If a direct summand $S$ of $T$ is in $m\left(\hat{R}_{p}\right) T$ then $S=m\left(\hat{R}_{p}\right) S$. Since $T$ (and even $\left.\hat{R}_{p}^{X}\right)$ is separated in the $m\left(\hat{R}_{p}\right)$-adic topology, this is impossible unless $S=0$. So by Lemma 1.1 we have a cover.

Any decomposition $T=T_{1} \oplus T_{2}$ gives one of $T / m\left(\hat{R}_{p}\right) T$, so by Lemma 1.3, $T_{1} \rightarrow T_{1} / m\left(\hat{R}_{p}\right) T_{1}$ is a flat cover. Uniqueness of covers and the computation above show that $T_{1}$ is also the completion of a free $\hat{R}_{p}$-module whose dimension is the same as that of $T_{1} / m\left(\hat{R}_{p}\right) T_{1}$ over $k(p)$. This means that a direct summand of the completion of a free module is again such.

If $k$ is a field and $\operatorname{Card}(X)=m<\infty$, the construction above shows that $k\left[\left[x_{1}, \ldots, x_{n}\right]\right]^{m} \rightarrow k^{m}$ is a flat cover. If $X$ is infinite, $k\left[\left[x_{1}, \ldots, x_{n}\right]\right]^{(X)} \rightarrow k^{(X)}$ is not even a precover. 


\section{Cotorsion modules.}

Definition. A module $M$ is said to be cotorsion if $\operatorname{Ext}^{1}(F, M)=0$ for all flat modules $F$. This generalizes the definition for abelian groups. It differs from Matlis' definition in his deep study [9] but his concern was with domains. It agrees with Fuchs' more general definition in [10] which deals with torsion theories.

Cotorsion groups are known to be uniquely, up to isomorphism, the direct sum of a divisible group, a cotorsion group which has no torsion free direct summands and a torsion free cotorsion group. The latter were classified by Harrison [13] as products $G=\Pi T_{p}$ over all primes $p$ where $T_{p}$ is a direct summand of $\hat{Z}_{p}^{X}$ for some $X$. Furthermore, $G$ is uniquely determined by the dimensions of $T_{p} / p T_{p}$ over $Z /(p)$. Our object is to extend this characterization to modules over any $R$.

We need the standard

LEMMA 2.1. If $E$ is an injective module and $M$ any module then $\operatorname{Hom}(M, E)$ is pure injective (and so cotorsion).

Proof. Pure injective means that if $0 \rightarrow S \rightarrow N$ is a pure injection, $H(N, \operatorname{Hom}(M, E)) \rightarrow \operatorname{Hom}(S, \operatorname{Hom}(M, E)) \rightarrow 0$ is surjective, but this is a result of the standard identity.

Note that if $M$ is $\hat{R}_{p}$-pure injective then $M$ is $R$-pure injective. Hence we see that any direct summand of a product of modules each of which is reflexive over some $\hat{R}_{p}$ is cotorsion (these modules also have flat covers).

LEMMA 2.2. If $\phi: F \rightarrow M$ is a flat cover then $\operatorname{Ker}(\phi)$ is cotorsion.

Proof. If $S \subset N$ is a submodule for some module $N$ with $N / S$ flat and $f$ : $S \rightarrow \operatorname{Ker}(\phi)$ is linear, let $N \oplus_{f} F$ be the amalgamated sum of $N$ and $F$ along $S$. Then $F \subset N \oplus_{f} F$ and $N \oplus_{f} F$ is flat. $\phi$ can be extended to a map $N \oplus_{f} F \rightarrow M$ which maps $N$ to 0 . If we complete

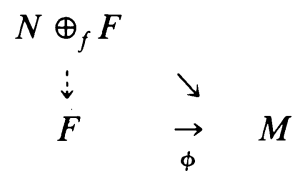

we can assume the vertical map induced the identity on $F$. But then the vertical map gives a map $N \rightarrow \operatorname{Ker}(\phi)$ which extends $f$. This clearly implies $\operatorname{Ker}(\phi)$ is cotorsion.

COROLlaRY. If $M$ is cotorsion then so is $F$.

Proof. $0=\operatorname{Ext}^{1}(G, \operatorname{Ker}(\phi)) \rightarrow \operatorname{Ext}^{1}(G, F) \rightarrow \operatorname{Ext}^{1}(G, M)$ is exact.

LEMMA 2.3. A flat module $F$ is cotorsion if and only if it is a direct summand of a module $\operatorname{Hom}\left(E, E^{\prime}\right)$ where $E$ and $E^{\prime}$ are injective.

Proof. A direct summand of $\operatorname{Hom}\left(E, E^{\prime}\right)$ is cotorsion by Lemma 2.1 and flat by Lemma 1.4. Conversely, if $F$ is flat, let $E$ be an injective generator. Then it is standard that $F \rightarrow \operatorname{Hom}(\operatorname{Hom}(F, E), E)$ is a pure injection (see [11 or 12]). $\operatorname{Hom}(F, E)$ is injective so $\operatorname{Hom}(\operatorname{Hom}(F, E), E)$ is flat. $F$ being cotorsion and a pure submodule makes it a direct summand. 
We now investigate the modules $\operatorname{Hom}\left(E, E^{\prime}\right)$. By Matlis [6], $E$ can be written $\oplus E(k(p))^{\left(X_{p}\right)}$ (over $\left.p\right)$ so

$$
\operatorname{Hom}\left(E, E^{\prime}\right) \cong \prod \operatorname{Hom}\left(E(k(p)), E^{\prime}\right)^{X_{p}} \cong \prod \operatorname{Hom}\left(E(k(p)), E^{\prime X_{p}}\right) ;
$$

hence we consider the modules $\operatorname{Hom}(E(k(p)), G)$ for $G$ injective. We have $\operatorname{Hom}(E(k(p)), G) \cong \operatorname{Hom}\left(E(k(p)) \oplus R_{p}, G\right) \cong \operatorname{Hom}\left(E(k(p)), \operatorname{Hom}\left(R_{p}, G\right)\right)$.

But $\operatorname{Hom}\left(R_{p}, G\right)$ is injective over $R_{p}$ so is a sum of $E(k(q))$ 's for $q \subset p$. But $\operatorname{Hom}(E(k(p)), E(k(q)))=0$ if $q \subsetneq p$. We conclude that

$$
\operatorname{Hom}(E(k(p)), G) \cong \operatorname{Hom}\left(E(k(p)), E(k(p))^{(Y)}\right)
$$

for some $Y$, i.e. it is the completion of a free $\hat{R}_{p}$-module with base indexed by $Y$.

We now have

THEOREM. For a module $F$ the following are equivalent:

(a) $F$ is a flat cover of some cotorsion module.

(b) $F$ is flat and cotorsion.

(c) $F \cong \Pi T_{p}$ (over $\left.p \in \operatorname{Spec}(R)\right)$ where $T_{p}$ is the completion of a free module over $R_{p}$.

Furthermore the decomposition in (c) is uniquely determined by the dimension of the free modules.

Proof. (a) $\Rightarrow$ (b) By the corollary to Lemma 2.2 .

(b) $\Rightarrow$ (c) We note that we have argued above that $F$ is a direct summand of such a product $\Pi T_{p}$. If $G=\Pi T_{p}$, then if $q \in \operatorname{Spec}(R)$ we have $q T_{p}=T_{p}$ if $q \not \subset p$ and $\cap q^{n} T_{p}=0$ if $q \subset p$. Hence $G^{\prime}=\cap q^{n} T_{p}=\Pi T_{p}$ for $p \not \subset q$, so $H=G / G^{\prime} \cong \Pi T_{p}$ for $p \subset q$. But then

$$
\bigcap_{p \subsetneq q}\left(\bigcap_{n} p^{n} H\right) \cong T_{q} .
$$

This means that given $G$ we can "recover" each $T_{p}$. The procedure commutes with direct sums, i.e. if $G=G_{1} \oplus G_{2}$ then we get an induced decomposition $T_{p}=\left(T_{p}\right)_{1}$ $\oplus\left(T_{p}\right)_{2}$ for each $p$ so that $G_{1} \cong \Pi\left(T_{p}\right)_{1}$. But as noted earler, $\left(T_{p}\right)_{1}$ is again the completion of a free module over $R_{p}$. This proves $(\mathrm{b}) \Rightarrow(\mathrm{c})$ and the last statement of the theorem.

For (c) $\Rightarrow$ (a), we already have that $T_{p} \rightarrow T_{p} / m\left(\hat{R}_{p}\right) T_{p}$ is a flat cover for each $p$ so $\Pi T_{p} \rightarrow \Pi T_{p} / m\left(\hat{R}_{p}\right) T_{p}$ is a flat precover with kernel $K=\Pi m\left(\hat{R}_{p}\right) T_{p}$. Let $F=\Pi T_{p}$ and suppose $S \subset K$ is a direct summand of $F$. If $q$ is such that $S \subset q F$ then $S=q S$, which implies that the projection of $S$ on $T_{q}$ is 0 (since $T_{q}$ is separated in the $q$-adic topology). Hence $S=0$ if $S \subset q F$ for all $q$. If not, let $q$ be maximal with $S \not \subset q F$. But if $q \not \subset p$, then $q T_{p}=T_{p}$. If $q \subsetneq p$ then, as above, the projection of $S$ on $T_{p}$ is 0 . Since $q T_{q}=m\left(\hat{R}_{q}\right) T_{q}$ we get $S \subset q F$, which is a contradiction.

\section{REFERENCES}

1. E. Enochs, Injective and flat covers, envelopes and resolvents, Israel J. Math. 39 (1981), 189-209.

2. __ Torsion free covering modules, Proc. Amer. Math. Soc. 14 (1963), 884-889. 
3. B. Banaschewski, On coverings of modules, Math. Nachr. 31 (1966), 57-71.

4. E. Enochs, Torsion free covering modules. II, Arch. Math. (Basel) 22 (1971), 37-52.

5. T. Ishikawa, On injective modules and flat modules, J. Math. Soc. Japan 17 (1965), 291-296.

6. E. Matlis, Injective modules over noetherian rings, Pacific J. Math. 8 (1958), 511-528.

7. P. Griffith, A representation theorem for complete local rings, J. Pure Appl. Algebra 7 (1976), 303-315.

8. L. Fuchs, Infinite abelian groups, Vol. 1, Academic Press, New York, 1970.

9. E. Matlis, Cotorsion modules, Mem. Amer. Math. Soc. 49 (1964).

10. L. Fuchs, Cotorsion modules over noetherian hereditary rings, Houston J. Math. 3 (1977), 33-46.

11. M. Raymond and L. Gruson, Critères de platitude et de projectivité, Invent. Math. 13 (1971), 1-89.

12. R. B. Warfield, Purity and algebraic compactness for modules, Pacific J. Math. 28 (1969), 699-719.

13. D. K. Harrison, Infinite abelian groups and homological methods, Ann. of Math. (2) 69 (1959), 366-391.

Department of Mathematics, University of Kentucky, LeXington, Kentucky 40506 